\title{
Propensity score matching confirms that primary surgery or neoadjuvant chemotherapy result in equivalent survival within a comprehensive cohort of patients with high-grade serous ovarian cancer
}

Dol:

10.1016/j.ygyno.2020.10.035

Document Version

Accepted author manuscript

Link to publication record in Manchester Research Explorer

Citation for published version (APA):

Edmondson, R., \& Hawarden, A. (2021). Propensity score matching confirms that primary surgery or neoadjuvant chemotherapy result in equivalent survival within a comprehensive cohort of patients with high-grade serous ovarian cancer. Gynecologic Oncology, 160(1), 24-31. https://doi.org/10.1016/j.ygyno.2020.10.035

\section{Published in:}

Gynecologic Oncology

\section{Citing this paper}

Please note that where the full-text provided on Manchester Research Explorer is the Author Accepted Manuscript or Proof version this may differ from the final Published version. If citing, it is advised that you check and use the publisher's definitive version.

\section{General rights}

Copyright and moral rights for the publications made accessible in the Research Explorer are retained by the authors and/or other copyright owners and it is a condition of accessing publications that users recognise and abide by the legal requirements associated with these rights.

\section{Takedown policy}

If you believe that this document breaches copyright please refer to the University of Manchester's Takedown Procedures [http://man.ac.uk/04Y6Bo] or contact uml.scholarlycommunications@manchester.ac.uk providing relevant details, so we can investigate your claim.

\section{OPEN ACCESS}


1

2

3

4

5 7

8

PROPENSITY SCORE MATCHING CONFIRMS THAT PRIMARY SURGERY OR NEOADJUVANT CHEMOTHERAPY RESULT IN EQUIVALENT SURVIVAL WITHIN A COMPREHENSIVE COHORT OF PATIENTS WITH HIGH-GRADE SEROUS OVARIAN CANCER

B. Russell ${ }^{1}$, A Hawarden ${ }^{1,2}$ M. Gee ${ }^{1,2}$, RJ. Edmondson ${ }^{1,2}$

1 Division of Cancer Sciences, Faculty of Biology, Medicine and Health, University of Manchester, Saint Mary's Hospital, Manchester, UK;

2Department of Obstetrics and Gynaecology, Manchester Academic Health Science Centre, Saint Mary's Hospital, Manchester University NHS Foundation Trust, Manchester Academic Health Science Centre; Level 5, Research, Oxford Road, Manchester, UK;

\section{Running Title:}

Key words: ovarian cancer, surgery, neoadjuvant chemotherapy, propensity score analysis

\section{Corresponding author}

RJ Edmondson

\section{Chair of Gynaecological Oncology}

Division of Cancer Sciences,

University of Manchester,

Manchester Academic Health Science Centre, St Mary's Hospital,

Research Floor,

Oxford Road,

Manchester,

M13 9WL, UK

richard.edmondson@manchester.ac.uk

Word count (excluding references): 2981

Total number of figures and tables: 6 figures $\& 1$ table 
35 Objective

36 Our objective was to investigate whether trial evidence showing that neoadjuvant chemotherapy is non inferior to primary surgery for the primary treatment of advanced ovarian cancer could be extrapolated to groups of patients that were not included in the trials.

39 Methods

40 Using a detailed retrospective cohort of all patients managed through a single tertiary hospital we 41 carried out a propensity score analysis, principal component analysis, and cox proportional hazard 42 analysis to compare survival in matched cohorts.

43 Results

44 A propensity score analysis showed that for at least $41 \%$ of all patients with advanced high-grade 45 serous cancer neoadjuvant chemotherapy is non inferior to primary surgery (median survival 46 primary surgery: 38 months, neoadjuvant chemotherapy: 35 months. $P=0.39$ ). However, principal 47 component analysis, supported by cox modelling, suggests that for some subgroups, including 48 patients with subdiaphragmatic nodal disease, primary surgery may be associated with improved 49 survival (HR 0.11, $\mathrm{Cl} 0.026-0.48)$.

50 Conclusions

51 We have shown that the findings of previous trials can be extrapolated to a wider population and 52 that statistical modelling can be used to identify groups or patients who benefit from specific 53 modalities of treatment. 
The majority of the 239,000 patients who are diagnosed worldwide each year with epithelial ovarian cancer (EOC) will present with advanced disease which has spread beyond the pelvis [1] .

Despite the widespread distribution of tumour deposits, surgery, originally defined by Griffiths over 40 years ago [2], remains a critical part of the treatment strategy for patients with advanced disease. The aim of surgery has always been to remove all macroscopic disease as this is associated with the highest survival rates [3].

Attempts to improve cytoreductive rates, and therefore by assumption survival, have focussed on comparing primary cytoreductive surgery (PCS) against a regimen of neoadjuvant chemotherapy (NACT) and delayed surgery. To date there have been two published randomised controlled trials [4, 5], and there are a further three trials underway or awaiting survival analysis [6-8] . Results have been combined in a pooled analysis [9] and a Cochrane review [10], and continue to show broadly equivalent outcomes.

In the two studies for which data is available [5, 7], recruitment was highly selective with low recruitment rates per centre per year in SCORPION (1.7 patients per centre per year) [7] and CHORUS (1.0 patients per centre per year) [5].

Further evidence of case selection for recruitment can be seen in the short survival times seen in both CHORUS and EORTC $55971[4,5]$. These may be a result of the population of included patients who were older, who had a poorer performance status, and who had higher stage tumors compared with other trials: Observational studies examining women over the age 65 years in the SEERMedicare database had median survivals that were similar to the EORTC and CHORUS trial participants [11] .' In addition, nearly $25 \%$ of the patients enrolled in the CHORUS trial received single-agent carboplatin instead of a platinum-based doublet in both the PCS and neoadjuvant arms further highlighting that these trials tended to include poorer prognosis groups.

Given these uncertainties extrapolation of the results to the whole population of patients with advanced EOC may be inappropriate [12]. Furthermore, although the trials, to date, have shown non inferiority to NACT there is no clear guidance on how to select optimal treatment for an individual patient [13] . An exploratory subset analysis of EORTC, which showed that patients with less extensive tumors ( $\leq 45 \mathrm{~mm}$ ) had better survival with PCS compared with NACT, likely suffers the same inclusion bias as the main trial itself [14] .

We therefore sought to answer two questions: To what proportion of cases do the results of the previous randomised trials apply and are there subgroups of patients who may benefit from one treatment pathway over another?

Methods

\section{Case Identification and Data Collection}

All cases of epithelial ovarian cancer referred to the multidisciplinary team (MDT) at Saint Mary's Hospital, Manchester, a tertiary referral centre, were included in the study. Cases were diagnosed on the basis of histology, either from an image guided biopsy or following primary resection. Only in cases where no histology was available was cytology used for diagnosis.

Following case identification, the dataset was limited to those cases with advanced disease (FIGO stage III and IV) who commenced active treatment. 
Treatment pathway was determined by members of the multidisciplinary team (MDT) following review of available histology, radiology, tumour markers and basic clinical characteristics. For the duration of the recruitment period, local guidance to clinicians was "Primary debulking surgery is the standard-of-care for patients of good performance status when complete or optimal cyto-reduction seems achievable. Neoadjuvant chemotherapy may also be considered if the prospects for optimal debulking at laparotomy are remote. In women with significant medical co-morbidity or whose performance status is poor, consideration should also be given at the MDT to recommending initial chemotherapy in place of primary surgery." Saint Mary's is a large tertiary referral centre seeing approximately 100 new cases of ovarian cancer each year. Patients are managed by a specialist multidisciplinary team comprising six surgical gynae oncologists and three medical oncologists. Ultimate treatment decision was made between oncologist and patient. All patient data were collected from the relevant electronic clinical health records by experienced gynae oncology fellows (AH \& MG). For each patient, data were collected according to a predefined data dictionary. Items selected for inclusion in the data dictionary were the result of an extensive literature search and included all items which were known to have both prognostic value and that were available in the clinical record. Morbidity data were retrieved from prospectively collected records generated during and after each admission.

The CT scan data were collected from electronic notes recorded at the MDT discussion. During the $\mathrm{MDT}$, a consultant radiologist would describe anatomical sites where disease could be detected from the CT scan and these sites were systematically recorded in the MDT notes. These notes were read, in conjunction with the formal CT report, by clinically trained members of the study team. There remains a lack of consensus upon an accurate way to assess tumour volume or distribution preoperatively. Therefore, the diagnostic CT scan reports, generated by specialist radiologists, were mined to generate a radiology score, based on presence or absence of disease in up to 31 anatomical sites, adapted from [15-17], supplementary table 1.

Patients were then classified into two treatment strata, those whose treatment pathway commenced with primary cytoreductive surgery (PCS) and those whose treatment pathway commenced with neoadjuvant chemotherapy (NACT), irrespective of whether those patients subsequently went on to have delayed surgery (subsequently referred to as treatment intention).

\section{Preliminary data interrogation}

Covariate balance was checked between the two treatment strata. For continuous and categorical variables, Mann-Whitney $U$ tests and chi square tests were used respectively. Log rank test was calculated from the Kaplan-Meier estimate to examine differences in the survival of those receiving PS and those receiving NACT.

\section{Propensity score matching}

Propensity score matching (PSM) was used to reduce bias in the survival estimates of the two treatment pathways. A logistic regression model was built using all available covariates available with the intention to treat as the target variable. Each patient's propensity was calculated using the generated model. 
The matchit package in R (GNU Affero General Public License v3) was used for the propensity matching. Calliper matching was used with a calliper size of 0.2 , as previously recommended [18]. After matching, the covariates were checked for balance between intention to treat arms to ensure there was no significant difference. A Kaplan-Meier estimate was used to check for a significant difference in the survival of patients stratified by intention to treat.

\section{Cox proportional hazard regression model.}

In order to check if the intention to treat was significant in the survival of our cohort, a Cox proportional hazard model was used. The model was built using all covariates described above. A concordance index (c-index) was generated to validate the predictive ability of the survival model [19].

\section{Principal component analysis}

In order to explore if groups of patients exist that may benefit from one treatment over another, principal component analysis (PCA) was used in conjunction with a k-nearest neighbour algorithm. Principal components were calculated from the scaled and centred covariates previously described. Principal components were plotted against each other in a 2D space. A hypothetical new "test patient" could thus be projected into the 2D space. The k-nearest neighbour algorithm was then used to find the closest patients in the 2D space for each treatment arm. A Cox proportional hazard model was built from the patients identified via the nearest neighbour process to compare survival between the treatment options. In this case $k=20$ was chosen, since it reliably resulted in the generation of Cox models with acceptable concordance.

In order to explore this idea for all patients, a search grid was created covering the 2D space. At each grid intersect location, the nearest neighbour and cox model procedure was repeated. If areas of significance were found, then loadings plots from the principal component analysis could be used to infer clinical characteristics of patients in this area. Heat maps of the 2D PCA space were created to visualise areas of significant differences in survival. The heatmap would only be coloured if $p<0.05$ at that grid intersect.

\section{Results}

\section{Case identification and Data collection}

475 patients with a diagnosis of epithelial ovarian cancer were referred to the Saint Mary's MDT between 2013 - 2017. Of those, 217 were defined as having stage 3 or 4 high-grade serous ovarian cancer. A further 22 cases were removed because of lacking any CT data, figure 1 . Due to a complete lack of $\mathrm{CT}$ data for these patients, they were removed from analysis since imputation 
methods would not be appropriate in this scenario. There was no relationship between absence of CT data and treatment strata precluding the presence of bias.

Treatment intention was defined as the initial treatment modality received. Any patient who received upfront primary debulking surgery was recorded as PCS, whilst those who received chemotherapy as their initial active treatment were recorded as NACT. 75/195 (38\%) had PCS as treatment intention while 120/195 (62\%) had NACT. Of the 120 NACT patients, 69 patients had delayed primary surgery, while 51 did not receive any surgery. The median follow up time of the cohort was 30.7 months (Range $0.07-81.2$ months). The rate of suboptimal cytoreduction (greater than $1 \mathrm{~cm}$ residual disease) for patients undergoing surgery during the study period was $8 \%$ for PCS and $10 \%$ for NACT patients.

\section{Preliminary data interrogation}

There were significant differences in covariates between patients treated with PCS and NACT, table 1. Specifically, patients treated with NACT tended to be older (median age 69 vs $65, p=0.008$ ), have a worse performance score $(47.4 \%$ PCS $>=2$ vs $20.3 \%, p<0.0005)$ and a higher disease burden as estimated in the CT findings, table 1.

Overall survival was also significantly higher (median OS, 56 months, range $0.1-77.2$ months) for patients in the PS group compared to those in the NACT group (median OS, 30 months, range 1.1 81.2 months), figure 2

\section{Propensity score matching}

Propensity score matching was used to generate a matched cohort. Propensity scores for each patient (representing the likelihood of a patient being allocated to primary surgery) showed differences in the distribution between patients allocated to NACT compared to patients having primary surgery. However, considerable overlap existed, suggesting that it would be possible to generate a matched cohort, figure $3 \mathrm{~A}$.

Using a calliper matching with a width of $0.2,80 / 195$ (41\%) of patients were able to be included in a matched cohort comprising 40 primary surgery patients and 40 NACT patients. Comparison of all covariates between treatment intentions was found to be non significant $(p>0.05)$ which indicates adequate matching, supplementary figure 2 . Figure $3 \mathrm{~B}$ shows the continuous variables stratified by treatment intention for the matched cohort. When comparing the well-matched cohorts of patients there was no difference in survival between patients in the primary surgery group (median survival 38 months, range $0.1-77.2$ months) compared to patients in the neoadjuvant group (median survival 35 months, range $2.2-81.2$ months) ( $p=0.39$, log rank test), figure $3 C$.

There was no significant difference in the number of patients with post - op complications (chi square, $p=0.7$ ) or length of stay after surgery (Mann-Whitney $U$ test $p=0.07$ ) between treatment intentions for the matched patients. 
Next, we sought to identify possible variables associated with adverse survival outcome. A Cox proportional hazard model was built using all 13 covariates. The resultant concordance index was 0.7 .

Although trending toward favouring PCS, treatment modality (primary surgery or neoadjuvant chemotherapy) was not a significant variable in the model with a hazard ratio of $0.62(0.37-1.0)$. Of the continuous variables, only younger age was significantly associated with survival. However, in relation to the imaging variables, the presence of subdiaphragmatic nodal disease and the presence of ascites were both associated with worse survival (HRs 1.98, $\mathrm{Cl} 1.3-3$ and 1.65, $\mathrm{Cl} 1.1-2.5$ respectivly). Figure 4 shows the forest plot of the Cox - proportional hazard model.

Finally we sought to identify variables associated with improved survival dependent upon treatment modality. A principal component analysis was carried out using the full dataset and all 13 variables. A 2D search space, comprising PC1 and PC2 was generated, figure 5a. Cox models were generated for each nearest neighbour matched cohort of 40 patients ( 20 primary surgery and 20 NACT). For each grid intersect where treatment intention was found to be significant $(p<0.05)$ in the cox regression model, the grid intersect was coloured according to the $p$ value, figure $5 a$.

Hazard ratios within this area favoured patients undergoing primary surgery.

The area around 0,0 in the principal component space was found to generate models where treatment intention was found to be highly significant. Inspection of the loading plot, figure $5 \mathrm{~b}$, indicated that this group of patients were likely matched in this area due to them presenting with subdiaphragmatic nodal disease.

Given that both the Cox Model and the principal component analysis appeared to show benefits to patients with subdiaphragmatic nodal disease being treated with primary surgery, the dataset was split in two on the condition of patients having subdiaphragmatic nodal disease.

248 This created two new datasets, ( $n=149$, absence of subdiaphragmatic nodal disease; $n=46$, presence 249 of subdiaphragmatic nodal disease). A Cox- proportional hazard model was generated for each of these two groups using all the previously described variables, excluding the subdiaphragmatic nodal disease variable. For the group of patient with subdiaphragmatic nodal disease, improved survival was associated with those patients undergoing primary surgery with a hazard ratio of $0.11(\mathrm{Cl} 0.026$ $-0.48, p=0.003)$. 
For patients where no subdiaphragmatic nodal disease was present, the intention to treat was not found to be significant in the Cox-proportional hazard model $(p=0.45)$. Figure 5 shows the adjusted survival curves for the two groups of patients, startified by the intention to treat.

\section{Discussion}

In this study we sought to answer two questions: To what proportion of cases do the results of the previous randomised trials apply and are there subgroups of patients who may benefit from one treatment pathway over another?

By including all cases referred to a single institution we can be confident in our finding that, for at least $41 \%$ of patients, there is no difference in survival whether they are treated with surgery or chemotherapy initially. This provides confidence that the findings from the randomised trials $[4,5]$ can be applied to a wider population than was included in the trials. It is possible that the actual percentage of patients to which this result applies is larger than $41 \%$ but our dataset prevented further matching.

Our secondary objective was to demonstrate that these methods could be used to identify potential subgroups of patients who would benefit from one form of treatment over another. Given our relatively small population there is a significant risk of statistical overfitting in the current study and we encourage caution in over interpreting these findings. Nevertheless our finding that patients with subdiaphragmatic nodal disease appear to have improved survival if they are treated with surgery first is interesting and worthy of further investigation. Indeed there is a biological plausibility to this finding, several authors suggest that nodal disease is a distinct entity compared to peritoneal disease [20] and indeed the FIGO staging system was changed to reflect this [21] with better outcomes for patients with nodal disease rather than intraperitoneal disease [22] . Although no studies have been done that compare treatment patterns in nodal disease, stratification of this subgroup should be considered in future trials.

Advanced ovarian cancer is unusual amongst solid tumours in that the predominant pattern of spread is via the transcoelomic route resulting in disseminated peritoneal disease. It is possible that nodal disease, which behaves in a more conventional metastatic way than peritoneal disease, requires the more conventional approach of surgical cytoreduction before chemotherapy, in contrast to peritoneal disease which can be treated with NACT.

Alternatively, large nodes will be removed completed at primary surgery whereas after NACT, where there has been a partial response and reduction in size of lymph nodes, involved nodes are not identified or removed and remain as sanctuary sites for recurrence [23].

The strength of our study is that it includes all patients referred to a single centre and therefore avoids the recruitment bias which has affected previous randomised trials. Indeed, ongoing surgical trials have narrowed the inclusion criteria even further [8] . This has limited the potential relevance of findings from these studies and will continue to engender controversy. We therefore took care to not only make this a comprehensive study but also to curate the data carefully and include variables that reflected patient factors and tumour factors. Assessing tumour load from CT scans is challenging 
[24] and is therefore not often included in studies. However given the likely importance of tumour burden in predicting outcome future studies should ensure careful curation of imaging data.

In this single centre study we have demonstrated that statistical techniques can be used to extrapolate results from randomised controlled trials. These techniques could now be used, with multi centre data, to identify and validate other subgroups of patients who would benefit from personalised algorithms.

301

Acknowledgements

Conflicts of Interest

$\mathrm{RB}, \mathrm{AW}, \mathrm{MG}$ state they have no conflicts of interest to declare. RJE has received consultancy funding from GSK \& Arquer Diagnostics

\section{Author Credit Statement}

Russell: Conceptualization; Data curation; Formal analysis; Investigation; Methodology; Resources; Software; Validation; Visualization; Roles/Writing - original draft; Writing - review \& editing.

Hawarden: Conceptualization; Data curation; Formal analysis; Investigation; Methodology; Resources; Validation; Visualization; Writing - review \& editing.

Gee: Conceptualization; Data curation; Writing - review \& editing.

Edmondson: Conceptualization; Data curation; Formal analysis; Funding acquisition; Investigation; Methodology; Project administration; Resources; Software; Supervision; Validation; Visualization; 
Table 1 -clinical characteristics

\begin{tabular}{|c|c|c|c|c|}
\hline & $\begin{array}{c}\text { Total } \\
\text { No. } 195\end{array}$ & $\begin{array}{c}\text { Primary } \\
\text { Surgery } \\
\text { No. } 75\end{array}$ & $\begin{array}{l}\text { NACT } \\
\text { No. } 120\end{array}$ & p-value \\
\hline Age & \multicolumn{3}{|c|}{$67.0(59.0-73.5) 65.0(55.5-69.5) 69.0(62.0-75.0)$} & 0.008 \\
\hline Stage & & & & 0.001 \\
\hline 3 & $147(75.4 \%)$ & $66(88.0 \%)$ & $81(67.5 \%)$ & \\
\hline 4 & $48(24.6 \%)$ & $9(12.0 \%)$ & $39(32.5 \%)$ & \\
\hline PerformanceStatus & & & & 0.0002 \\
\hline High & $70(36.8 \%)$ & $15(20.3 \%)$ & $55(47.4 \%)$ & \\
\hline Low & $120(63.2 \%)$ & $59(79.7 \%)$ & $61(52.6 \%)$ & \\
\hline \multicolumn{5}{|l|}{ Diagnostic blood markers } \\
\hline CA125 kU/L & $792(272-2000)$ & $491(200-1300)$ & $1017(298-2073)$ & 0.031 \\
\hline Albumin & $37(33-40)$ & $37(35-40)$ & $37(32-41)$ & 0.93 \\
\hline Haemoglobin (g/L) & $122(111-133)$ & $130(118-136)$ & $117(108-127)$ & $<0.0001$ \\
\hline Platelets & $395(298-518)$ & $343(278-435)$ & $442(325-551)$ & 0.0004 \\
\hline Neutrophil & $5.6(4.5-7.5)$ & $5.5(4.4-7.1)$ & $5.7(4.7-8.2)$ & 0.35 \\
\hline Lymphocyte & $1.4(1.1-2.1)$ & $1.7(1.2-2.3)$ & $1.4(1.1-1.8)$ & 0.027 \\
\hline \multicolumn{5}{|l|}{ CT disease ${ }^{1}$} \\
\hline Non resectable sites ${ }^{2}$ & 75 (38.5\%) & $13(17.3 \%)$ & $62(51.7 \%)$ & $<0.0001$ \\
\hline Bowel disease & $66(33.8 \%)$ & $13(17.3 \%)$ & $53(44.2 \%)$ & $<0.0001$ \\
\hline Resectable sites $^{3}$ & $164(84.1 \%)$ & $54(72.0 \%)$ & $110(91.7 \%)$ & 0.0005 \\
\hline $\begin{array}{l}\text { Subdiaphragmatic } \\
\text { nodal }^{4}\end{array}$ & $46(23.6 \%)$ & $16(21.3 \%)$ & $30(25.0 \%)$ & 0.61 \\
\hline Ascites & $91(46.7 \%)$ & $34(45.3 \%)$ & $57(47.5 \%)$ & 0.88 \\
\hline
\end{tabular}
aggregated, see supplementary data 2 sites generally considered to be unresectable including porta hepatis disease, multiple hepatic metastases, multiple pulmonary metastases and mediastinal nodal disease 3extra pelvic, intra peritoneal disease 4 retroperitoneal nodal disease below the level of the diaphragm 
Figure 1 - consort diagram demonstrating patient flow

Figure 2. Kaplan-Meier curves of the whole cohort $(\mathrm{N}=195)$ stratified by intention to treat. The primary surgery group have significantly better survival than the NACT group of patients.

Figure 3. Propensity score matching. (A) Box and whisker plot identifies significant differences in propensity to be selected for PCS compared to NACT, indicating there are significant differences in the populations receiving the treatments. (B) box and whisker plots show 133 cases could be matched and show good matching of each of the continuous variables for the 133 cases which were matched. There are no significant differences in any of the variables between the treatment arms. (C) Kaplan Meier curves of the matched cohort stratified by intention to treat. There is no significant difference in survival between the two treatment arms for the matched cohort $(n=133)$.

347 Figure 4.Forest plot of the Cox proportional hazard model. Only age, disease in the subdiaphragmatic nodes (Nodal_CT) and presence of ascites were significantly associated with survival.

Figure 5 - (A) Two dimensional principle component analysis plot using full dataset and 13 variables per data point. Each data point is coloured (red for chemotherapy, blue for surgery). Plot is overlaid to show differences in survival with grey areas highlighting areas within plot which are associated with significantly improved survival for patients having primary surgery. No areas are identified which favour NACT, and (B) radar plot showing relative weighting of variables in dimension 1 and 2 of the PCA shown in (A) (see supplementary data for definitions). Plot identifies subdiaphragmatic nodal disease as major determinant of significance seen in $(A)$

Figure 6. Adjusted survival curves stratified by intention to treat. (A) shows the curves for patients without nodal disease. (B) shows the curves for patients with nodal disease. For patients without nodal disease, there is no significant difference in the survival between primary surgery and NACT. For patients with nodal disease, the intention to treat is highly significant. 


\section{Author Contribution Statement}

Russell: Conceptualization; Data curation; Formal analysis; Investigation; Methodology; Resources; Software; Validation; Visualization; Roles/Writing - original draft; Writing - review \& editing.

Hawarden: Conceptualization; Data curation; Formal analysis; Investigation; Methodology; Resources; Validation; Visualization; Writing - review \& editing.

Gee: Conceptualization; Data curation; Writing - review \& editing.

Edmondson: Conceptualization; Data curation; Formal analysis; Funding acquisition; Investigation; Methodology; Project administration; Resources; Software; Supervision; Validation; Visualization; Roles/Writing - original draft; Writing - review \& editing.

\section{References}

1. Ferlay, J., et al., GLOBOCAN 2012 v1.0, Cancer Incidence and Mortality Worldwide: IARC CancerBase No. 11. International Agency for Research on Cancer, 2013.

2. Griffiths, C., Surgical resection of tumor bulk in the primary treatmetn of ovarian carcinoma. Nat Cancer Inst Monogr, 1975. 42: p. 101-104.

3. Bristow, R.E., et al., Survival Effect of Maximal Cytoreductive Surgery for Advanced Ovarian Carcinoma During the Platinum Era: A Meta-Analysis

10.1200/JCO.20.5.1248. J Clin Oncol, 2002. 20(5): p. 1248-1259.

4. Vergote, I., et al., Neoadjuvant Chemotherapy or Primary Surgery in Stage IIIC or IV Ovarian Cancer. New England Journal of Medicine, 2010. 363(10): p. 943-953.

5. Kehoe, S., et al., Primary chemotherapy versus primary surgery for newly diagnosed advanced ovarian cancer (CHORUS): an open-label, randomised, controlled, non-inferiority trial. Lancet, 2015.

6. Onda, T., et al., Comparison of treatment invasiveness between upfront debulking surgery versus interval debulking surgery following neoadjuvant chemotherapy for stage III/IV ovarian, tubal, and peritoneal cancers in a phase III randomised trial: Japan Clinical Oncology Group Study JCOG0602. European Journal of Cancer, 2016. 64: p. 22-31.

7. Fagotti, A., et al., Phase III SCORPION trial (ID number: NCT01461850) in epithelial ovarian cancer patients with high tumor load receiving PDS versus NACT: An interim analysis on perioperative outcome. Gynecologic Oncology, 2015. 138: p. 1-2.

8. Reuss, A., et al., TRUST: Trial of Radical Upfront Surgical Therapy in advanced ovarian cancer (ENGOT ov33/AGO-OVAR OP7). Int J Gynecol Cancer, 2019. 29(8): p. 1327-1331.

9. Vergote, I., et al., Neoadjuvant chemotherapy versus debulking surgery in advanced tuboovarian cancers: pooled analysis of individual patient data from the EORTC 55971 and CHORUS trials. Lancet Oncol, 2018. 19(12): p. 1680-1687.

10. Coleridge, S.L., et al., Chemotherapy versus surgery for initial treatment in advanced ovarian epithelial cancer. Cochrane Database Syst Rev, 2019. 2019(10).

11. Thrall, M.M., et al., Neoadjuvant chemotherapy in the Medicare cohort with advanced ovarian cancer. Gynecologic Oncology, 2011. 123(3): p. 461-466.

12. Charlton, B.G., Participants in research: routine extrapolation of randomised controlled trials is absurd. Bmj, 2005. 331(7508): p. 109-10. 
13. Wright, A.A., et al., Neoadjuvant Chemotherapy for Newly Diagnosed, Advanced Ovarian Cancer: Society of Gynecologic Oncology and American Society of Clinical Oncology Clinical Practice Guideline. J Clin Oncol, 2016. 34(28): p. 3460-73.

14. van Meurs, H.S., et al., Which patients benefit most from primary surgery or neoadjuvant chemotherapy in stage IIIC or IV ovarian cancer? An exploratory analysis of the European Organisation for Research and Treatment of Cancer 55971 randomised trial. Eur J Cancer, 2013. 49(15): p. 3191-201.

15. Rutten, M.J., et al., Predicting surgical outcome in patients with International Federation of Gynecology and Obstetrics stage III or IV ovarian cancer using computed tomography: a systematic review of prediction models. Int J Gynecol Cancer, 2015. 25(3): p. 407-15.

16. Son, H.M., et al., Preoperative prediction of suboptimal resection in advanced ovarian cancer based on clinical and CT parameters. Acta Radiol, 2017. 58(4): p. 498-504.

17. Suidan, R.S., et al., A multicenter assessment of the ability of preoperative computed tomography scan and CA-125 to predict gross residual disease at primary debulking for advanced epithelial ovarian cancer. Gynecol Oncol, 2017. 145(1): p. 27-31.

18. Lunt, M., Selecting an Appropriate Caliper Can Be Essential for Achieving Good Balance With Propensity Score Matching. American Journal of Epidemiology, 2013. 179(2): p. 226-235.

19. Koziol, J.A. and Z. Jia, The concordance index $C$ and the Mann-Whitney parameter $\operatorname{Pr}(X>Y)$ with randomly censored data. Biom J, 2009. 51(3): p. 467-74.

20. Berek, J.S., Lymph node-positive stage IIIC ovarian cancer: a separate entity? Int J Gynecol Cancer, 2009. 19 Suppl 2: p. S18-20.

21. Duska, L.R. and E.C. Kohn, The new classifications of ovarian, fallopian tube, and primary peritoneal cancer and their clinical implications. Annals of Oncology, 2017. 28: p. viiii-viii12.

22. Gasimli, K., et al., Lymph Node Involvement Pattern and Survival Differences of FIGO IIIC and FIGO IIIA1 Ovarian Cancer Patients After Primary Complete Tumor Debulking Surgery: A 10Year Retrospective Analysis of the Tumor Bank Ovarian Cancer Network. Ann Surg Oncol, 2016. 23(4): p. 1279-86.

23. Pereira, E.R., et al., The lymph node microenvironment and its role in the progression of metastatic cancer. Semin Cell Dev Biol, 2015. 38: p. 98-105.

24. Rockall, A.G., et al., Repeatability of quantitative FDG-PET/CT and contrast-enhanced CT in recurrent ovarian carcinoma: test-retest measurements for tumor FDG uptake, diameter, and volume. Clin Cancer Res, 2014. 20(10): p. 2751-60. 